Bull. Austral. Math. Soc.

Vol. 55 (1997) [207-218]

\title{
COMPLETE ENDOMORPHISMS OF THE LATTICE OF PSEUDOVARIETIES OF FINITE SEMIGROUPS
}

\author{
Norman R.Reilly and ShuHua Zhang \\ The main result establishes that the mapping $\mathbf{V} \rightarrow \mathbf{V} \cap \mathbf{W}(\mathbf{V} \in \mathcal{L}(\mathbf{F}))$ is a \\ complete endomorphism of the lattice $\mathcal{L}(\mathbf{F})$ of pseudovarieties of finite semigroups \\ for certain particular pseudovarieties $W$, including the pseudovariety of bands.
}

\section{INTRODUCTION}

In a series of recent papers, certain techniques that have proved valuable in the study of the lattice of varieties of completely regular semigroups (see Pastijn [12], Polák [20], Petrich and Reilly [16, 17]) involving the existence of certain complete congruences have been extended, first to the lattice of e-varieties of regular semigroups (Reilly and Zhang [24]) and then to the study of the lattice $\mathcal{L}(\mathbf{F})$ of pseudovarieties of finite semigroups (Auinger, Hall, Reilly and Zhang [4]). In particular, it is shown in [4] that there exists a family of complete congruences of the form:

$$
\mathbf{U} \Theta_{\mathbf{P}} \mathbf{V} \quad \Longleftrightarrow \mathbf{P} \circ \mathbf{U}=\mathbf{P} \circ \mathbf{V} \quad(\mathbf{U}, \mathbf{V} \in \mathcal{L}(\mathbf{F}))
$$

where $\circ$ denotes the Mal'cev product and $\mathbf{P}$ is any one of the eight pseudovarieties $\mathbf{N}, \mathbf{L Z} \circ \mathbf{N}, \mathbf{R Z} \circ \mathbf{N}, \mathbf{R B} \circ \mathbf{N}, \mathbf{G} \circ \mathbf{N}, \mathbf{L G} \circ \mathbf{N}, \mathbf{R G} \circ \mathbf{N}$ or $\mathbf{C S} \circ \mathbf{N}$. This introduces an interesting interplay between the lattice theoretic structure of $\mathcal{L}(\mathbf{F})$ and the Mal'cev product and leads naturally to the study of operators on $\mathcal{L}(\mathbf{F})$ of the form $\mathbf{V} \rightarrow \mathbf{P} \circ \mathrm{V}$. This study was pursued by the present authors in [25]. Operators on $\mathcal{L}(F)$ of the form $\mathbf{V} \rightarrow \mathbf{V} \cap \mathbf{W}$, for certain special pseudovarieties $\mathbf{W}$, such as the pseudovariety of groups, bands, et cetera also play an important role in the study of $\mathcal{L}(F)$ and are the main focus of this paper.

In the main result, in Section 3, we show that the mapping

$$
\mathbf{V} \rightarrow \mathbf{V} \cap \mathbf{W} \quad(\mathbf{V} \in \mathcal{L}(\mathbf{F}))
$$

is a complete endomorphism of $\mathcal{L}(\mathbf{F})$ for a certain family of pseudovarieties $\mathbf{W}$, the most interesting of which is, perhaps, the pseudovariety of bands. It is also shown that the mapping

$$
\mathbf{V} \longrightarrow \mathbf{V} \cap \mathbf{C R}
$$

Received 28th March, 1996

This work was supported, in part by NSERC Grant 4044.

Copyright Clearance Centre, Inc. Serial-fee code: 0004-9729/97 \$A2.00+0.00. 
is a complete endomorphism on the sublattice of $\mathcal{L}(F)$ consisting of those pseudovarieties in which the union of the subgroups of any member is a subsemigroup. These observations will be used by the authors in a subsequent paper to study the operators of the form $\mathbf{V} \rightarrow \mathbf{P} \circ \mathbf{V}$ referred to above.

\section{Preliminaries}

All semigroups considered in this paper are finite. We denote by $\mathbf{F}$ the class of all finite semigroups. A pseudovariety is a class of finite semigroups which is closed under taking finite direct products, subsemigroups and homomorphic images. For elementary notions and results on semigroups and pseudovarieties, the reader is referred to the books of Almeida [2], Clifford and Preston [5], Eilenberg [6], Howie [9] and Pin [19]. For basic information on lattices, the reader is referred to Grätzer [7].

Let $S \in \mathbf{F}$. For $x \in S$ (respectively, $X \subseteq S$ ) we denote by $\langle x\rangle$ (respectively, $\langle X\rangle$ ) the subsemigroup of $S$ generated by $x$ (respectively, $X$ ). We denote by $E(S)$ the set of all idempotents of $S$.

Since $\mathcal{J}=D$ in any finite semigroup (see Howie [9, Proposition II.1.5]), we shall always write $\mathcal{J}$ in preference to $\mathcal{D}$.

A semigroup $S$ is a completely regular semigroup if it is a union of groups, equivalently, if each $\mathcal{H}$-class of $S$ is a group. A completely regular semigroup for which $E(S)$ forms a subsemigroup is an orthogroup and a completely regular semigroup for which $\mathcal{H}$ is a congruence is a cryptogroup. If $S$ is both an orthogroup and a cryptogroup then it is an orthocryptogroup.

In a completely regular semigroup $S$, we use the following notation. If $x \in S$, then $x^{-1}$ is the inverse of $S$ in the maximal subgroup of $S$ containing $x$. In addition, let $x^{0}=x x^{-1}$.

For any nonempty subclass $\mathbf{A}$ of $\mathbf{F}$, we shall denote by $\langle\mathbf{A}\rangle$ the pseudovariety generated by $\mathbf{A}$. In the case $\mathbf{A}=\{S\}$, where $S \in \mathbf{F}$, we simply write $\langle S\rangle$ instead of $\langle\{S\}\rangle$.

For any pseudovariety $\mathbf{U}$ of finite semigroups, the class of all subpseudovarieties of $\mathbf{U}$ forms a complete lattice under inclusion, and is denoted by $\mathcal{L}(\mathrm{U})$. In particular, $\mathcal{L}(\mathbf{F})$ is a complete lattice under inclusion (see Eilenberg [6] or Pin [19]).

The concepts of implicit operations and pseudoidentities are important tools in the study of pseudovarieties. We refer the reader to Almeida [2] and Pin [19] for their basic properties and for a discussion of Reiterman's Theorem [26] which states that every pseudovariety is uniquely determined by the pseudoidentities that it satisfies.

One implicit operation of particular importance is the operation

$$
x \rightarrow x^{\omega} \quad(x \in S \in \mathbf{F}),
$$


where $x^{\omega}$ denotes the unique idempotent in $\langle x\rangle$. We also write $x^{\omega+1}=x^{\omega} x=x x^{\omega}$ and $x^{\omega-1}=\left(x^{\omega+1}\right)^{-1}$, the inverse of $x^{\omega+1}$ in the group kernel of $\langle x\rangle$.

We list some pseudovarieties of finite semigroups that will be used in this paper:

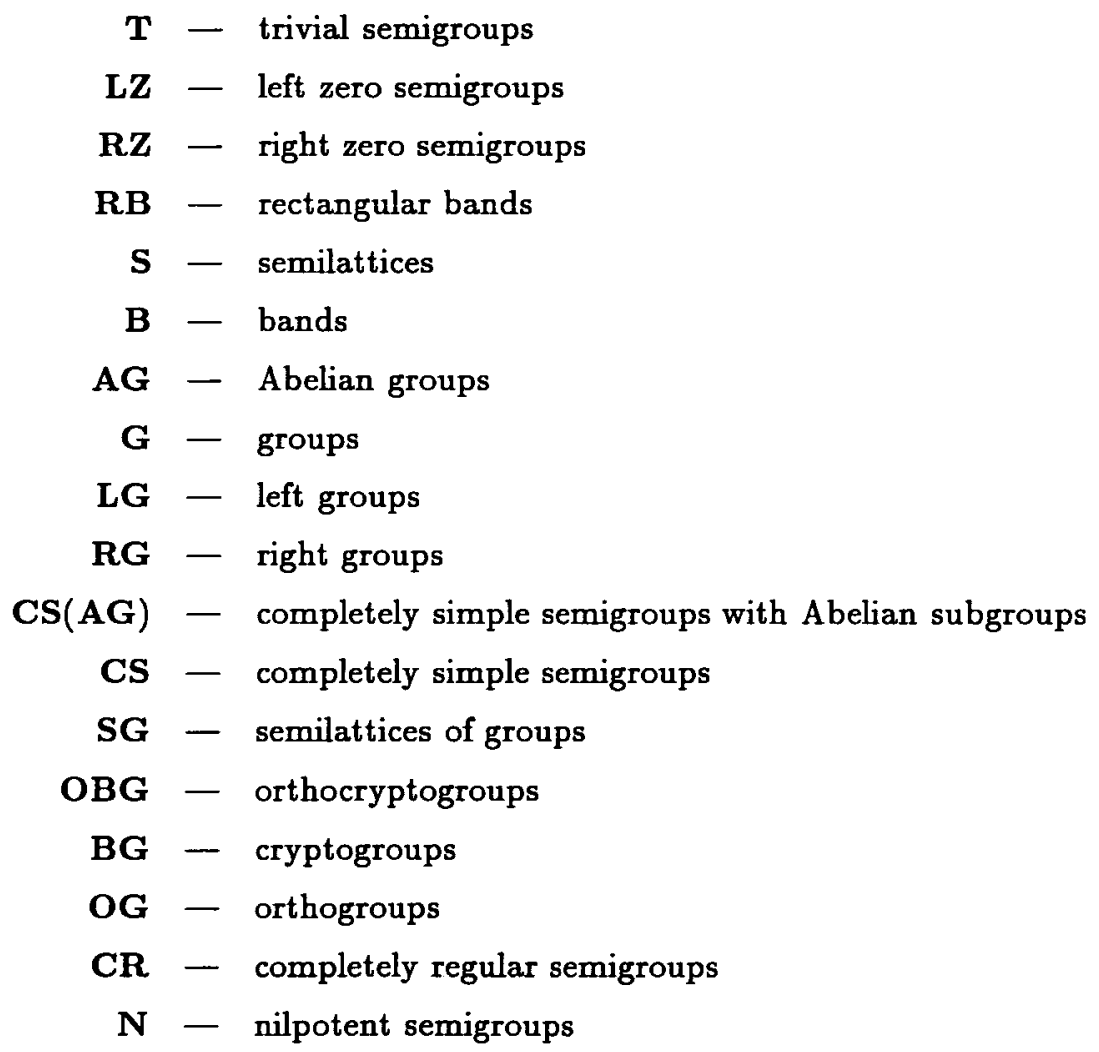

The reader is referred to Almeida [2], Eilenberg [6], Howie [9], Pin [19], Hall and Jones [8] and Petrich and Reilly [18] for some well-known relationships among the above listed pseudovarieties, such as $\mathbf{S G}=\mathbf{S} \vee \mathbf{G}, \mathbf{O B G}=\mathbf{G} \vee \mathbf{B}$.

We introduce an operator $L$ on $\mathcal{L}(\mathbf{F})$ as follows. For any $U \in \mathcal{L}(\mathbf{F})$, let

$$
L \mathrm{U}=\{S \in \mathrm{F} \mid e S e \in \mathrm{U} \text { for all } e \in E(S)\}
$$

Equivalently, $L U$ consists of those finite semigroups $S$ all of whose submonoids are in U. By Eilenberg [6, Section V.1], $L U \in \mathcal{L}(\mathbf{F})$.

The following technical lemma, due to Krohn, Rhodes and Tilson [3, Fact 2.1 in Chapter 7], will be needed in the sequel.

Lemma 2.1. Let $S, T \in \mathrm{F}$ and $\varphi: S \longrightarrow T$ be a surjective homomorphism. Let $J_{2}$ be a $\mathcal{J}$-class of $T$ and $J_{1}$ be a minimal $\mathcal{J}$-class (in the $\mathcal{J}$-class ordering) of $S$ 
contained in $J_{2} \varphi^{-1}$. Then

(i) $J_{1} \varphi=J_{2}$;

(ii) $J_{1}$ is regular if and only if $J_{2}$ is regular.

Another well-known fact about complete congruences on a complete lattice that is of considerable utility, due to Pastijn and Petrich [14, Lemma 4.12], is the following:

LEMMA 2.2. Let $\theta$ be an equivalence relation on the complete lattice $L$ such that each $\theta$-class $x \theta$ is a bounded interval $x \theta=\left[x_{\theta}, x^{\theta}\right]$. Then $\theta$ is a complete congruence if and only if for all $x, y \in L, x \leqslant y$ implies $x_{\theta} \leqslant y_{\theta}$ and $x^{\theta} \leqslant y^{\theta}$.

\section{SOME COMPLETE ENDOMORPHISMS OF $\mathcal{L}(\mathrm{F})$}

In this section we shall show that some mappings induced by taking intersection with subpseudovarieties are complete endomorphisms of $\mathcal{L}(\mathbf{F})$. We shall require some preparation.

Lemma 3.1. [7, Theorem III.2.2] Let $L$ be a lattice and $a \in L$. Then the following are equivalent:

(a) The mapping

$$
\chi_{a}: x \longrightarrow a \wedge x \quad(x \in L)
$$

is an endomorphism of $L$;

(b) The binary relation $\Theta_{a}$ on $L$ defined by

$$
(x, y) \in \Theta_{a} \quad \Longleftrightarrow \quad a \wedge x=a \wedge y
$$

is a congruence relation on $L$.

Lemma 3.2. Let $U \in \mathcal{L}(\mathbf{F})$, and let $\chi_{\mathrm{U}}$ and $\Theta_{\mathrm{U}}$ be defined as in Lemma 3.1. Then the following statements are equivalent:

(a) $\chi_{\mathrm{U}}$ is an endomorphism of $\mathcal{L}(\mathbf{F})$;

(b) $\Theta_{\mathrm{U}}$ is a complete congruence on $\mathcal{L}(\mathbf{F})$;

(c) $\chi_{\mathrm{U}}$ is a complete endomorphism of $\mathcal{L}(\mathrm{F})$.

Proof: (a) $\Rightarrow(\mathrm{b})$. By Lemma 3.1, $\Theta_{\mathrm{U}}$ is a congruence on $\mathcal{L}(\mathrm{F})$. To see that $\Theta_{\mathbf{U}}$ is a complete congruence, we let $\mathbf{W}^{\Theta_{\mathbf{U}}}=\{S \in \mathrm{F} \mid\langle S\rangle \cap \mathbf{U} \subseteq \mathrm{W}\}$ for each $\mathbf{W} \in \mathcal{L}(\mathbf{F})$. Let $\mathbf{W} \in \mathcal{L}(\mathbf{F})$. It is clear from the definition that $\mathbf{W}^{\Theta_{\mathbf{U}}}$ is closed under taking subsemigroups and homomorphic images. Since, by the hypothesis,

$$
\begin{aligned}
\langle S \times T\rangle \cap \mathbf{U} & =(\langle S\rangle \vee\langle T\rangle) \cap \mathbf{U} \\
& =(\langle S\rangle \cap \mathbf{U}) \vee(\langle T\rangle \cap \mathbf{U}),
\end{aligned}
$$


it follows that $\mathbf{W}^{\Theta} \mathbf{U}$ is also closed under taking finite direct products. Hence $\mathbf{W}^{\Theta^{U}} \in$ $\mathcal{L}(\mathbf{F})$.

Let $\mathbf{W} \in \mathcal{L}(\mathbf{F})$. Since $\mathbf{W} \subseteq \mathbf{W}^{\Theta} \mathbf{U}$ and $\mathbf{W}^{\Theta} \mathbf{U} \cap \mathbf{U}=\mathbf{W} \cap \mathbf{U}$, it follows that [WחU, $\left.\mathbf{W}^{\Theta_{\mathbf{U}}}\right] \subseteq \mathbf{W} \Theta_{\mathbf{U}}$. On the other hand, if $\mathbf{V} \in \mathcal{L}(\mathbf{F})$ and $\mathbf{V} \in \mathbf{W} \Theta_{\mathbf{U}}$, then $\mathbf{V} \cap \mathbf{U}=\mathbf{W} \cap \mathbf{U}$ and $\mathbf{V}^{\Theta_{\mathbf{U}}} \subseteq \mathbf{W}^{\Theta_{\mathbf{U}}}$, so that $\mathbf{V} \in\left[\mathbf{W} \cap \mathbf{U}, \mathbf{W}^{\Theta_{\mathbf{U}}}\right]$. Hence $\mathbf{W} \Theta_{\mathbf{U}}=\left[\mathbf{W} \cap \mathbf{U}, \mathbf{W}^{\Theta_{\mathbf{U}}}\right]$. It is now clear that $\Theta_{U}$ satisfies the conditions in Lemma 2.2, and hence $\Theta_{U}$ is a complete congruence on $\mathcal{L}(\mathbf{F})$.

(b) $\Rightarrow$ (c). It is clear from the definition that $\chi_{U}$ is a complete $\wedge$-endomorphism of $\mathcal{L}(\mathbf{F})$. Let $\left\{\mathbf{W}_{\alpha}\right\}_{\alpha \in A}$ be any family of members of $\mathcal{L}(\mathbf{F})$. For each $\alpha \in A$, since $\mathbf{W}_{\boldsymbol{\alpha}} \cap \mathbf{U}=\left(\mathbf{W}_{\boldsymbol{\alpha}} \cap \mathbf{U}\right) \cap \mathbf{U}$, we have $\mathbf{W}_{\boldsymbol{\alpha}} \Theta_{\mathbf{U}}\left(\mathbf{W}_{\boldsymbol{\alpha}} \cap \mathbf{U}\right)$, so that by (b),

$$
\bigvee_{\alpha \in A} \mathbf{w}_{\alpha} \Theta_{\mathbf{U}} \bigvee_{\alpha \in A}\left(\mathbf{w}_{\boldsymbol{\alpha}} \cap \mathbf{U}\right)
$$

By the definition of $\Theta_{U}$, we get

$$
\begin{aligned}
\left(\bigvee_{\alpha \in A} \mathbf{w}_{\boldsymbol{\alpha}}\right) \cap \mathrm{U} & =\left(\bigvee_{\boldsymbol{\alpha} \in A}\left(\mathbf{w}_{\boldsymbol{\alpha}} \cap \mathrm{U}\right)\right) \cap \mathbf{U} \\
& =\bigvee_{\boldsymbol{\alpha} \in A}\left(\mathbf{w}_{\boldsymbol{\alpha}} \cap \mathbf{U}\right),
\end{aligned}
$$

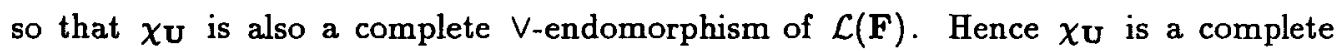
endomorphism of $\mathcal{L}(\mathbf{F})$.

(c) $\Rightarrow$ (a). Obvious.

For any $\mathrm{U}, \mathrm{V} \in \mathcal{L}(\mathbf{F})$, the Mal'cev product of $\mathrm{U}$ and $\mathrm{V}$ is

$$
\begin{gathered}
\mathrm{U} \circ \mathrm{V}=\{S \in \mathrm{F} \mid \\
\text { there exists a congruence } \rho \text { on } S \text { with } \\
\text { (i) } S / \rho \in \mathrm{V} \text { and (ii) } a \rho \in \mathrm{U} \text { for all } \\
\text { idempotent } \rho \text {-classes } a \rho\} .
\end{gathered}
$$

In particular, if $\mathbf{U}, V \in \mathcal{L}(\mathbf{C R})$, we let $\mathbf{U} \circ_{\mathbf{C R}} \mathbf{V}=(\mathbf{U} \circ \mathbf{V}) \cap \mathbf{C R}$. In general, $\mathbf{U} \circ \mathbf{V}$ is not a pseudovariety (see [11]).

In [13], F. Pastijn established the analogue of Polák's Theorem (see [20]) for pseudovarieties of finite completely regular semigroups. Define relations $K$ and $T$ on $\mathcal{L}(\mathbf{C R})$ by

$$
\mathrm{U} K \mathrm{~V} \quad \Longleftrightarrow \quad \mathbf{R B} \circ_{\mathrm{CR}}(\mathrm{U} \vee \mathrm{S})=\mathbf{R B} \circ_{\mathrm{CR}}(\mathrm{V} \vee \mathrm{S})
$$

and

$$
\mathrm{U} T \mathbf{V} \quad \Longleftrightarrow \quad \mathbf{G}_{\mathrm{CR}} \mathrm{U}=\mathbf{G} \mathrm{o}_{\mathrm{CR}} \mathrm{V}
$$


LEMMA 3.3. [13]

(i) $K$ and $T$ are complete congruences on $\mathcal{L}(\mathbf{C R})$;

(ii) $K \cap T=\iota$, the identity relation.

Since $K$ and $T$ are complete congruences on $\mathcal{L}(\mathbf{C R})$ it follows that the $K$ - and $T$-classes are intervals. For any $\mathrm{V} \in \mathcal{L}(\mathbf{C R})$, we write:

$$
\mathrm{V} K=\left[\mathrm{V}_{K}, \mathrm{~V}^{K}\right] \quad \text { and } \quad \mathrm{V} T=\left[\mathrm{V}_{T}, \mathrm{~V}^{T}\right] .
$$

From the standard methods for completely regular semigroups (see Hall-Jones [8], Pastijn-Trotter [15], Polák [20], Petrich-Reilly [17] and Reilly [21]) we have:

LEMma 3.4. Let $\mathrm{V} \in \mathcal{L}(\mathrm{CR})$. Then

(i) $\mathbf{V}^{K}=\mathbf{R B} \circ_{\mathrm{CR}}(\mathrm{V} \vee \mathrm{S})$ and $\mathrm{V}^{T}=\mathbf{G} \circ_{\mathrm{CR}} \mathrm{V}$;

(ii) $\mathrm{V}^{K} \vee \mathrm{V}^{T}=\mathrm{V}^{K T} \cap \mathrm{V}^{T K}$.

The particular instance of Lemma 3.4 that we are interested in occurs when $\mathbf{V}=$ RB. Then

$$
\begin{gathered}
\mathbf{R B}^{K}=\mathbf{B}, \quad \mathbf{R B}^{T}=\mathbf{C S}, \\
\mathbf{B}^{T}=\mathbf{B G}, \quad \mathbf{C S}^{K}=L(\mathbf{O G}) \cap \mathbf{C R},
\end{gathered}
$$

so that

$$
\begin{aligned}
\mathbf{C S} \vee \mathbf{B} & =\mathbf{R B}^{T} \vee \mathbf{R B}^{K} \\
& =\mathbf{R B}^{T K} \cap \mathbf{R B} \mathbf{B}^{K T} \\
& =L(\mathbf{O G}) \cap \mathbf{B G} \\
& =L(\mathbf{O B G}) \cap \mathbf{C R} .
\end{aligned}
$$

As in [7], an element $a$ in a lattice $L$ is neutral if the mapping

$$
x \longrightarrow(x \wedge a, x \vee a)
$$

is a monomorphism of $L$ onto a subdirect product of (a] and [a), where (a] and [a) denote the ideal and filter of $L$, respectively, generated by $a$.

Lemma 3.5. [7, Theorem III.2.6] Let $L$ be a modular lattice and $a \in L$. Then the following statements are equivalent:

(a) $a$ is neutral in $L$;

(b) The mapping

$$
\chi_{a}: x \longrightarrow a \wedge x \quad(x \in L)
$$




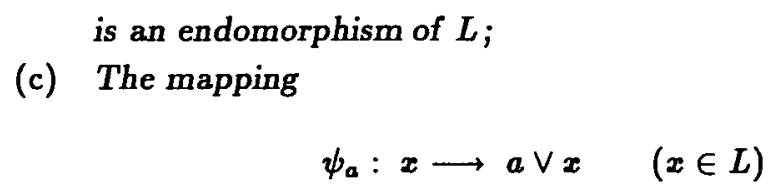

is an endomorphism of $L$.

In [10], Jones showed that every variety of normal bands is neutral in the lattice $\mathcal{L}_{v}(\mathbf{C R})$ of all varieties of completely regular semigroups. With the assistance of Polák's representation of $\mathcal{L}_{v}(\mathbf{C R})$ (see [20]), Pastijn established the modularity of $\mathcal{L}_{v}(\mathbf{C R})$ in [12] (see also [18]). The first author [22] and Trotter [27] then established the neutrality of many varieties in $\mathcal{L}_{v}(\mathbf{C R})$. The modularity of the lattice $\mathcal{L}(\mathbf{C R})$ of pseudovarieties of finite completely regular semigroups was established by F. Pastijn in [13]. The discussion of neutral elements of $\mathcal{L}_{v}(\mathbf{C R})$ in [22] and [27] then carries over with few changes to $\mathcal{L}(\mathbf{C R})$. In this way we obtain

LEMMA 3.6 .

(a) Every element of $\mathcal{L}(\mathbf{B} \vee \mathbf{C S}(\mathbf{A G}))$ is neutral in $\mathcal{L}(\mathbf{C R})$;

(b) The set $(B] \cup\{\mathbf{G}, \mathbf{C S}, \mathbf{O G}\}$ generates a sublattice of neutral elements of $\mathcal{L}(\mathbf{C R})$;

(c) If $\mathrm{V} \in \mathcal{L}(\mathbf{C R})$ is neutral in $\mathcal{L}(\mathbf{C R})$, then so is $\mathbf{V}^{K}$.

As an analogue of $[8$, Corollary 5.7] for pseudovarieties, we have

LEMMA 3.7. The mappings

$$
\mathbf{V} \longrightarrow(\mathrm{V} \cap \mathbf{C S}, \mathbf{V} \cap \mathbf{B}), \quad(\mathrm{U}, \mathbf{W}) \longrightarrow \mathbf{U} \vee \mathbf{W}
$$

are mutually inverse isomorphisms between $[\mathrm{RB}, L(\mathrm{OG}) \cap \mathbf{B G}]$ and $[\mathrm{RB}, \mathrm{CS}] \times$ $[\mathbf{R B}, B]$.

Proof: Since $\mathrm{CS}$ and $\mathrm{B}$ are both neutral in $\mathcal{L}(\mathbf{C R})$ by Lemma 3.6 and $L(\mathrm{OG}) \cap$ $\mathbf{B G}=\mathbf{C S} \vee \mathbf{B}$, the result follows from purely lattice theoretic considerations (see Petrich and Reilly [18, Theorem 7.5]).

Let

$$
\mathrm{DCh}=\{S \in \mathbf{C R} \mid S / \mathcal{J} \text { is a chain }\}
$$

In [23], the first author studied the varieties of completely regular semigroups generated by members of $\mathbf{D C h}$. Since all the relevant examples in [23] are finite, the next result carries over to pseudovarieties.

LEMMA 3.8.

(a) For all $\mathbf{V} \in \mathcal{L}(\mathbf{B}), \mathbf{V}=\langle\mathbf{V} \cap \mathbf{D C h}\rangle$.

(b) There exist $\mathrm{V} \in \mathcal{L}(\mathbf{C R})$ such that $\mathrm{V} \neq\langle\mathrm{V} \cap \mathrm{DCh}\rangle$. 
Trivially, for any $\mathrm{V} \in \mathcal{L}(\mathrm{CS})$, we have

$$
\mathrm{V}=\mathrm{V} \cap \mathbf{D C h} .
$$

The next result enlarges the class of pseudovarieties $\mathrm{V}$ for which it is known that

$$
\mathbf{V}=\langle\mathbf{V} \cap \mathbf{D C h}\rangle \text {. }
$$

Proposition 3.9. Let $\mathbf{V} \in \mathcal{L}(L(\mathbf{O G}) \cap \mathbf{B G})$. Then $\mathbf{V}=\langle\mathbf{V} \cap \mathbf{D C h}\rangle$.

Proof: By Lemma 3.7, there exist $U \in \mathcal{L}(\mathbf{C S})$ and $\mathbf{W} \in \mathcal{L}(\mathbf{B})$ such that $\mathrm{V}=$ $\mathbf{U} \vee \mathbf{W}$. Since $\mathbf{U}=\mathbf{U} \cap \mathbf{D C h}$ and $\mathbf{W}=\langle\mathbf{W} \cap \mathbf{D C h}\rangle$, it follows that $\mathbf{V}=\langle\mathbf{V} \cap \mathbf{D C h}\rangle$.

Lemma 3.10. Let $S \in \mathrm{F}, T \in \mathrm{DCh}$ and $\varphi: S \rightarrow T$ be a surjective homomorphism. Then there exists a subsemigroup $R$ of $S$ such that $R \in \mathrm{DCh}$ and $\left.\varphi\right|_{R}$ is surjective.

Proof: Since $S$ is finite, so also is $T$ and we may list the $\mathcal{J}$-classes of $T$ as $D_{1}, \ldots, D_{n}$ where, since $T \in \mathrm{DCh}$, we may assume that

$$
D_{1}>D_{2}>\ldots>D_{n}
$$

in the natural partial order of the $\mathcal{J}$-classes.

For each $\alpha=1,2, \ldots, n$, there exists by Lemma 2.1 a $\mathcal{J}$-class $C_{\alpha}$ in $S$ that is the least $\mathcal{J}$-class in the $\mathcal{J}$-class order within $D_{\alpha} \varphi^{-1}$ and for which $C_{\alpha} \varphi=D_{\alpha}$. Since $D_{\alpha}$ is a subsemigroup of $T$ it follows that $C_{\alpha}$ must be a subsemigroup of $S$. Thus $C_{\alpha}$ is completely simple. For $\alpha>\beta$ we have

$$
D_{\alpha} D_{\beta} D_{\alpha} \subseteq D_{\beta},
$$

so that

$$
C_{\alpha} C_{\beta} C_{\alpha} \subseteq D_{\beta} \varphi^{-1} \text {. }
$$

But $C_{\beta}$ is the least $\mathcal{J}$-class in $D_{\beta} \varphi^{-1}$ whence we must have

$$
C_{\alpha} C_{\beta} C_{\alpha} \subseteq C_{\beta} .
$$

It follows easily from this that $R=\bigcup_{\alpha=1}^{n} C_{\alpha}$ is a completely regular subsemigroup of $S$ with $\mathcal{J}$-classes $C_{1}, \ldots, C_{n}$ that are linearly ordered:

$$
C_{1}>C_{2}>\ldots>C_{n}
$$

Thus $R \in \mathbf{D C h}$ and, since $C_{\alpha} \varphi=D_{\alpha}$, it follows that $\left.\varphi\right|_{R}$ is surjective. 
Lemma 3.11. Let $\mathrm{U}, \mathrm{V} \in \mathcal{L}(\mathrm{F})$ and let $S \in \mathrm{DCh}$ be such that $S \in \mathrm{U} \vee \mathrm{V}$. Then

$$
S \in\langle\mathrm{U} \cap \mathrm{DCh}\rangle \vee(\mathrm{V} \cap \mathrm{DCh}) .
$$

Proof: By the hypothesis, there exist $U \in \mathbf{U}, V \in \mathbf{V}$, a subsemigroup $P$ of $U \times V$ and a surjective homomorphism $\varphi: P \rightarrow S$. By Lemma 3.10, there must be a subsemigroup $R$ of $P$ such that $R \in \mathrm{DCh}$ and $\left.\varphi\right|_{R}$ is surjective. There is no loss in generality in assuming that $P=R$ and that $R$ is a subdirect product of $U$ and $V$. This implies that $U$ and $V$ are both homomorphic images of $R$, which clearly implies that $U, V \in \mathrm{DCh}$. This establishes the claim.

We are now ready to prove the main result of this paper.

Theorem 3.12. Let $\mathrm{W} \in \mathcal{L}(\mathrm{CR})$ have the following properties:

(i) $\quad \mathbf{W}$ is neutral in $\mathcal{L}(\mathbf{C R})$;

(ii) $\mathbf{V} \in \mathcal{L}(\mathbf{W})$ implies that $\mathrm{V}=\langle V \cap \mathbf{D C h}\rangle$.

Then the mapping

$$
\chi_{\mathbf{w}}: \mathbf{U} \longrightarrow \mathbf{U} \cap \mathbf{W} \quad(\mathbf{U} \in \mathcal{L}(\mathbf{F}))
$$

is a complete endomomorphism of $\mathcal{L}(\mathbf{F})$.

Proof: By Lemma 3.2, it sufficies to show that $\chi_{\mathbf{W}}$ is an endomorphism of $\mathcal{L}(\mathrm{F})$. So let $\mathrm{U}, \mathrm{V} \in \mathcal{L}(\mathrm{F})$ and $S \in((\mathrm{U} \vee \mathrm{V}) \cap \mathrm{W}) \cap \mathrm{DCh}$. By Lemma 3.11,

$$
S \in(\langle\mathbf{U} \cap \mathbf{D C h}\rangle \vee\langle\mathbf{V} \cap \mathbf{D C h}\rangle) \cap \mathbf{W}
$$

where, since $\mathbf{W}$ is neutral in $\mathcal{L}(\mathbf{C R})$.

$$
\begin{aligned}
(\langle\mathbf{U} \cap \mathbf{D C h}\rangle \vee\langle\mathbf{V} \cap \mathbf{D C h}\rangle) \cap \mathbf{W} & =(\langle\mathbf{U} \cap \mathbf{D C h}\rangle \cap \mathbf{W}) \vee(\langle\mathbf{V} \cap \mathbf{D C h}\rangle \cap \mathbf{W}) \\
& \subseteq(\mathbf{U} \cap \mathbf{W}) \vee(\mathbf{V} \cap \mathbf{W}) .
\end{aligned}
$$

By part (ii) of the hypothesis, it follows that

$$
(\mathbf{U} \vee \mathbf{V}) \cap \mathbf{W} \subseteq(\mathbf{U} \cap \mathbf{W}) \vee(\mathbf{V} \cap \mathbf{W})
$$

The reverse containment holds trivially. Hence $\chi_{\mathrm{w}}$ is an endomorphism of $\mathcal{L}(F)$.

By combining Lemma 3.6 and Proposition 3.9 with Theorem 3.12, we obtain

Corollary 3.13. For each $W \in \mathcal{L}(B \vee C S(A G)) \cup\{G, C S, B \vee G, B \vee C S\}$, the mapping

$$
\chi_{\mathbf{W}}: \mathbf{U} \longrightarrow \mathrm{U} \cap \mathbf{W} \quad(\mathbf{U} \in \mathcal{L}(\mathbf{F}))
$$


is a complete endomorphism of $\mathcal{L}(\mathrm{F})$.

Corollary 3.13 includes previously known examples such as $\mathbf{W}=\mathbf{G}$ or $\mathbf{C S}$ or $\mathbf{S}$ (see [1], [2] and [4]) and some new examples such as $\mathbf{W}=\mathbf{B}$ or $\mathbf{B} \vee \mathrm{CS}(\mathbf{A G})$ or $\mathbf{B} \vee \mathbf{G}$ or $\mathbf{B} \vee \mathbf{C S}$.

We conclude this section with an observation on the mapping $\chi_{\mathrm{CR}}$ induced by taking intersections with CR.

Let $S \in \mathrm{F}$. Then $S$ is called group closed if $G_{r}(S)$, the set of all group elements of $S$, is a subsemigroup (which then is clearly completely regular) of $S$. Denote the class of all finite group closed semigroups by GC. By [25, Lemma 3.2], GC $=\left[x^{\omega+1} y^{\omega+1}=\right.$ $\left(x^{\omega+1} y^{\omega+1}\right)^{\omega+1}$ ] so that $\mathrm{GC}$ is a pseudovariety.

LEMMA 3.14. The mapping

$$
\chi_{\mathbf{C R}}: \mathbf{U} \rightarrow \mathbf{U} \cap \mathbf{C R}
$$

is a complete endomorphism of $\mathcal{L}(\mathbf{G C})$ onto $\mathcal{L}(\mathbf{C R})$.

Proof: In view of Lemma 3.2, it suffices to show that $\chi_{\mathbf{C R}}$ is a homomorphism of $\mathcal{L}(\mathbf{G C})$ onto $\mathcal{L}(\mathbf{C R})$. So let $\mathbf{U}, \mathbf{V} \in \mathcal{L}(\mathbf{G C})$, and let $S \in(\mathbf{U} \vee \mathbf{V}) \cap \mathbf{C R}$. Then there exists $U \in \mathbf{U}, V \in \mathrm{V}$, a subsemigroup $R$ of $U \times V$ and a surjective homomorphism $\varphi: R \rightarrow S$. It follows easily from Lemma 2.1 that the restriction of $\varphi$ to $G_{r}(R)$ is a homomorphism of $G_{r}(R)$ onto $S$, since $R \in \mathbf{G C}$. On the other hand, we have

$$
\begin{aligned}
G_{r}(R) & \subseteq G_{r}(U \times V) \\
& =G_{r}(U) \times G_{r}(V) \\
& \in(\mathbf{U} \cap \mathbf{C R}) \vee(\mathbf{V} \cap \mathbf{C R}),
\end{aligned}
$$

whence $S \in(\mathbf{U} \cap \mathbf{C R}) \vee(\mathbf{V} \cap \mathbf{C R})$. It follows that

$$
(\mathbf{U} \vee \mathbf{V}) \cap \mathbf{C R} \subseteq(\mathbf{U} \cap \mathbf{C R}) \vee(\mathbf{V} \cap \mathbf{C R}) .
$$

The reverse containment holds trivially. Hence $\chi_{\mathrm{CR}}$ is a complete homomorphism of $\mathcal{L}(\mathbf{G C})$ onto $\mathcal{L}(\mathbf{C R})$.

The above result automatically leads us to the following question: Is $\chi_{\mathrm{CR}}$ a complete endomorphism of $\mathcal{L}(\mathbf{F})$ ?

For any $\mathbf{U} \in \mathcal{L}(\mathbf{C S})$, the class

$$
D \mathrm{U}=\{S \in \mathrm{F} \mid \text { all regular } \mathcal{J} \text {-classes of } S \text { belong to } \mathrm{U}\}
$$

is always a pseudovariety. We refer the reader to [2] for the discussion of various examples. Note that our notation differs from that in [2] slightly, since we write $D R B$ where Almeida would write DA and we write DCS where Almeida would write DS. 
It follows from the discussion in $[\mathbf{2 3}]$ that the pseudovariety $\langle\mathbf{D C h}\rangle$ is a proper subpseudovariety of CR. A slightly larger class than DCh is

$$
\operatorname{DCh}(D C S)=\{S \in D C S \mid \text { the regular } \mathcal{J} \text {-classes of } S \text { form a chain }\} .
$$

For any words $p, q$, let

$$
[p, q]=p^{\omega+1} q^{\omega+1} p^{\omega-1} q^{\omega-1} .
$$

For any variables $x, y, w, z$ let

$$
a=(x y)^{\omega} x, \quad b=(w z)^{\omega} w \quad \text { and } \quad e=\left(a^{\omega} b^{\omega}\right)^{\omega}
$$

and

$$
u=\left[\left[e x e, e x^{2} e\right],\left[e y e, e y^{2} e\right]\right] .
$$

Then the elements of $\operatorname{DCh}(D C S)$ satisfy the pseudoidentity $u^{2}=u$. Since $D C S$ does not satisfy this pseudoidentity, it follows that $\langle\mathbf{D C h}(D \mathrm{CS})\rangle$ is a proper subpseudovariety of $D$ CS.

Note AdDed IN PROof. Part (a) of Lemma 3.8 also appears in "Linear Semigourps of Idempotents" by I.O. Koryakov, Investigations in modern algebra (Ed. L.N. Sherrin), Mathematical Proceedings of the Ural University, Sverdlousk KK (1978), 54-109 (Russian).

\section{REFERENCES}

[1] J. Almeida, 'On finite simple semigroups', Proc. Edinburgh Math. Soc. 34 (1991), 204-215.

[2] J. Almeida, Finite semigroups and universal algebra (World Scientific, Singapore, 1994).

[3] M. Arbib (ed.), The algebraic theory of machines, languages and semigroups (Academic Press, New York, 1968).

[4] K. Auinger, T.E. Hall, N.R. Reilly and S. Zhang, 'Congruences on the lattice of pseudovarieties of finite semigroups', Internat J. Algebra Comput. (to appear).

[5] A.H. Clifford and G.B. Preston, The algebraic theory of semigroups, Math. Surveys No. 7 (Amer. Math. Soc., Providence, RI, Vol. I, 1961; Vol. II, 1967).

[6] S. Eilenberg, Automata, languages and machines B (Academic Press, New York, 1976).

[7] G. Grätzer, General lattice theory (Birkhaūser, Basel, 1978).

[8] T.E. Hall and P.R. Jones, On the lattice of varieties of bands of groups, Pacific J. Math. $\theta 1$ (1980), 327-337.

[9] J.M. Howie, An introduction to semigroup theory (Academic Press, London, 1976).

[10] P.R. Jones, 'On the lattice of varieties of completely regular semigroups', J. Austral. Math. Soc. 35 (1983), 227-235.

[11] P.R. Jones, 'Mal'cev products of varieties of completely regular semigroups', J. Austral. Math. Soc. 42 (1987), 227-246. 
[12] F. Pastijn, 'The lattice of completely regular semigroup varieties', J. Austral. Math. Soc. 48 (1990), 24-42.

[13] F. Pastijn, 'Pseudovarieties of completely regular semigroups', Semigroup Forum 42 (1991), 1-46.

[14] F. Pastijn and M. Petrich, 'The congruence lattice of a regular semigroup', J. Pure Appl. Algebra 53 (1988), 93-123.

[15] F. Pastijn and P.G. Trotter, 'Lattices of completely regular semigroup varieties', Pacific J. Math. 119 (1985), 191-214.

[16] M. Petrich and N.R. Reilly, 'Semigroups generated by certain operators on varieties of completely regular semigroups', Pacific J. Math. 132 (1988), 151-175.

[17] M. Petrich and N.R. Reilly, 'Operators related to E-disjunctive and fundamental completely regular semigroups', J. Algebra 134 (1990), 1-27.

[18] M. Petrich and N.R. Reilly, 'The modularity of the lattice of varieties of completely regular semigroups and related representations', Glasgow Math. J. 32 (1990), 137-152.

[19] J.-E. Pin, Varieties of formal languages (North Oxford Academic Press, London, 1986).

[20] L. Polák, 'On varieties of completely regular semigroups I', Semigroup Forum 32 (1985), 97-123; II 36 (1987), 253-284; III 37 (1988), 1-30.

[21] N.R. Reilly, 'Varieties of completely regular semigroups', J. Austral. Math. Soc. 38 (1985), 372-393.

[22] N.R. Reilly, 'Completely regular semigroups', in Lattices, semigroups and universal algebra, (J. Almeida et al., Editors) (Plenum Press, New York, 1990), pp. 225-242.

[23] N.R. Reilly, 'Bounds on the variety generated by completely regular syntactic monoids from finite prefix codes', Theoret. Comput. Sci. 134 (1994), 189-208.

[24] N.R. Reilly and S. Zhang, 'Congruence relations on the lattice of existence varieties of regular semigroups', J. Algebra 178 (1995), 733-759.

[25] N.R. Reilly and S. Zhang, 'Operators on the lattice of pseudovarieties of finite semigroups', Semigroup Forum (to appear).

[26] J. Reiterman The Birkhoff theorem for finite algebras, Algebra Universalis 14 (1982), 1-10.

[27] P.G. Trotter, 'Subdirect decompositions of the lattice of varieties of completely regular semigroups', Bull. Austral. Math. Soc. 39 (1989), 343-351. 